\title{
New approach to Forecasting Agro-based statistical models
}

\author{
Muhammad Akram \\ Department of Epidemiology and preventive Medicine, Monash University, \\ The Alfred centre, Melbourne, Victoria-3004, Australia \\ Muhammad.Akram@monash.edu \\ M. Ishaq Bhatti \\ Department of Economics and Finance, LaTrobe University \\ Melbourne, Victoria-3086, Australia \\ i.bhatti@latrobe.edu.au \\ Muhammad Ashfaq \\ Institute of Agricultural and Resource Economincs \\ University of Agriculture, Faisalabad, Pakistan \\ ashfaq9@hotmail.com \\ Asif Ali Khan \\ Office of Research, Innovation and Commercialization, \\ University of Agriculture, Faisalabad, Pakistan \\ asifpbg@uaf.edu.pk
}

Received 15 May 2015

Accepted 28 June 2016

\begin{abstract}
This paper uses various forecasting methods to forecast future crop production levels using time series data for four major crops in Pakistan: wheat, rice, cotton and pulses. These different forecasting methods are then assessed based on their out-of-sample forecast accuracies. We empirically compare three methods: BoxJenkins' ARIMA, Dynamic Linear Models (DLM) and exponential smoothing. The best forecasting models are selected from each of the methods by applying them to various agricultural time series in order to demonstrate the usefulness of the models and the differences between them in an actual application. The forecasts obtained from the best selected exponential smoothing models are then compared with those obtained from the best selected classical Box-Jenkins ARIMA models and DLMs using various forecast accuracy measures.
\end{abstract}

Keywords: forecast; exponential smoothing; ARIMA; dynamic linear model; forecast accuracy measure.

2000 Mathematics Subject Classification: 62M10, 37M10

\section{Introduction}

The ultimate objective of any forecasting method, such as ES or ARIMA, is to provide precise future predictions. Many new forecasting methods have become available over the last few decades, 
and researchers and practitioners use various different sets of methods to conduct their empirical forecasting comparisons. For example, the major finding of [16], and [14], who used thousands of time series, was that simple methods, such as simple exponential smoothing and damped trend methods perform as well or in many cases better than those that are more statistically sophisticated ones like ARIMA models. The flexibility of exponential smoothing is demonstrated by its ability to provide forecasts for the complete taxonomy of [19], as extended by [11] and [22].

It is important to evaluate forecast accuracy using genuine forecasts. That is, it is invalid to look at how well a model fits the historical data; the accuracy of forecasts can only be determined by considering how well a model performs on new data that were not used when fitting the model. When choosing models, it is common to use a portion of the available data for fitting, and use the rest of the data (out-of-sample data) for testing the model [11]. Then the testing data can be used to measure how well the model is likely to forecast on new data.

The aim of this paper is to assess different forecasting methods based on their out-of-sample forecast accuracies. We compare various methods, such as Box-Jenkins' ARIMA technique, dynamic linear models [20], and the exponential smoothing method, by applying them to some real time series data in order to demonstrate the differences between the models in an actual application. The rest of the paper is arranged as follows.

A brief theoretical background of various forecasting methods is given in Section 2. The various forecast accuracy measures are defined in Section 3. The three methods under discussion are then compared by applying them to various agricultural production time series in Section 4. The final section contains concluding remarks.

\section{Theoretical background}

\subsection{Exponential Smoothing}

Exponential smoothing (ES) methods, that were first proposed in the late 1950s [4], have since been among the most widely used forecasting procedures in industry and commerce, particularly in production planning, inventory control, sales forecasting, production scheduling and operations management. There are a variety of methods that fall into the ES family, each having the property that forecasts are weighted combinations of past observations. That is, forecasts produced using exponential smoothing methods are weighted averages of past observations, with the weights decaying exponentially as the observations get older. In other words, the more recent the observation the higher the associated weight.

ES can be applied to time series data, either for smoothing the data or for producing forecasts. The basic idea behind ES is to construct an exponentially weighted moving average (EWMA) for each component of the time series, namely the level, trend and seasonality. The weight and length of each component are determined through the smoothing parameters of the components, namely $\alpha$ for level, $\beta$ for trend and $\gamma$ for seasonality (for detail, see [17]).

\subsubsection{Modeling framework}

The state space framework offers a statistical rationale in support of the ES models, that are based on capturing and smoothing the existing structural components of a time series, namely the level, trend and seasonality. The framework involves 30 different models ( 15 with additive errors and 15 with multiplicative errors). Each model consists of a measurement equation that describes the observed 
data and some transition equations that describe how the unobserved components or states (level, trend, seasonal) change over time. Hence these are referred to as state space models. Each model is denoted by its error, trend and seasonal components, following the procedure of [10], in order to allow for easy cross-referencing of the models. They code any exponential smoothing model as a triplet ETS (error, trend, seasonality). The following shorthand is used: $\mathrm{N}$ for none, A for additive, $A_{d}$ for additive damped, $M$ for multiplicative and $M_{d}$ for multiplicative damped. For example, AAN refers to a model with additive errors, additive trend and no seasonality. Model ANN gives forecasts that are equivalent to simple exponential smoothing, model AAN underlies Holt's linear method, and the additive Holt-Winters' method is obtained by AAA. Table 1, taken from [11], shows the 15 models with additive errors.

Table 1. Models with additive errors in modelling framework

\begin{tabular}{|ll|ccc|}
\hline \multirow{2}{*}{$\begin{array}{c}\text { Trend } \\
\text { Component }\end{array}$} & \multicolumn{3}{c|}{ Seasonal Component } \\
$\mathrm{N}$ & (None) & $\mathrm{N}$ & $\mathrm{A}$ & $\mathrm{M}$ \\
& $(\mathrm{None})$ & (Additive) & (Multiplicative) \\
\cline { 2 - 5 } $\mathrm{A}$ & (Additive) & $\mathrm{NN}$ & $\mathrm{NA}$ & $\mathrm{NM}$ \\
$A_{d}$ & (Additive damped) & $A_{d} \mathrm{~N}$ & $A_{d} \mathrm{~A}$ & $A_{d} \mathrm{M}$ \\
$\mathrm{M}$ & (Multiplicative) & $\mathrm{MN}$ & $\mathrm{MA}$ & $\mathrm{MM}$ \\
$M_{d}$ & (Multiplicative damped) & $M_{d} \mathrm{~N}$ & $M_{d} \mathrm{~A}$ & $M_{d} \mathrm{M}$ \\
\hline
\end{tabular}

\subsubsection{State space models}

The state space models enable easy calculations of the likelihood, and provide facilities for the computation of prediction intervals for each model. For each of their methods, [11] proposed two state space models with a single source of error, following the general approach of [18]. The two state space formulations correspond to the additive error and multiplicative error cases, and give equivalent point forecasts but different prediction intervals and likelihoods. The general framework, given by [18], involves a state vector $x_{t}$ and state space equations of the form

$$
\begin{aligned}
& y_{t}=h\left(x_{t-1}\right)+k\left(x_{t-1}\right) \varepsilon_{t} \\
& x_{t}=f\left(x_{t-1}\right)+g\left(x_{t-1}\right) \varepsilon_{t},
\end{aligned}
$$

where $\left\{\varepsilon_{t}\right\}$ is a Gaussian white noise process with mean zero and variance $\sigma^{2}$. Define $e_{t}=k\left(x_{t-1}\right) \varepsilon_{t}$ and $\mu_{t}=h\left(x_{t-1}\right)$; then, $y_{t}=\mu_{t}+e_{t}$. The model with additive errors is written as $y_{t}=\mu_{t}+\varepsilon_{t}$, so, in this case, $k\left(x_{t-1}\right)=1$. The model with multiplicative errors is written as $y_{t}=\mu_{t}\left(1+\varepsilon_{t}\right)$. Thus, $k\left(x_{t-1}\right)=\mu_{t}$ for this model and $\varepsilon_{t}=e_{t} / \mu_{t}=\left(y_{t}-\mu_{t}\right) / \mu_{t}$, and hence, $\varepsilon_{t}$ is a relative error for the multiplicative model.

Equation (2.1) is called the measurement equation, and equation (2.2) is known as the transition equation. The term $h\left(x_{t-1}\right)$ describes the effect of the past on $y_{t}$. The term $f\left(x_{t-1}\right)$ shows the effect of the past on the current state $x_{t}$. The term $g\left(x_{t-1}\right) \varepsilon_{t}$ shows the unpredictable change in $x_{t}$, and the vector $g$ determines the extent of the effect of the innovation on the state. All of the exponential smoothing methods of [11] can be written in the form of equations (2.1) and (2.2). The underlying equations are given in ( [10], Table $2.2 \& 2.3$ ). The only difference between the additive error 
and multiplicative error models is in the observation equation (2.1): multiplicative error models are obtained by replacing $\varepsilon_{t}$ in additive error models with $\mu_{t} \varepsilon_{t}$.

ES methods are arguably the most widely applied forecasting methods. They can also be regarded as a sub-case of the ARIMA framework ( [3]), but they are often preferred to ARIMA models due to their simplicity, robustness and accuracy. However, [11] showed that ES is in fact a general class of state space models that can provide optimal forecasts for a broader set of cases than ARIMA. In the opposite direction, an ARIMA model can also be put in the form of a linear state space model. The ES method has the advantage that its algorithm can be implemented relatively easily in a matrix language such as R [21] and/or 'Matlab'. This algorithm is implemented in the R package forecast [7].

\subsection{Box-Jenkins ARIMA models}

ARIMA models provide another approach to time series forecasting. ARIMA is an acronym for AutoRegressive Integrated Moving Average model (integration in this context is the reverse of differencing). Exponential smoothing and ARIMA models are the two most widely-used approaches to time series forecasting, and provide complementary approaches to the problem. While exponential smoothing models were based on a description of trend and seasonality in the data, ARIMA models aim to describe the autocorrelations in the data. Since a vast literature on ARIMA methodology is available (see for example [3]), we provide a brief description here.

\subsubsection{Autoregressive models}

In a multiple regression model, we forecast the variable of interest using a linear combination of predictors. In an autoregression model, we forecast the variable of interest using a linear combination of past values of the variable. The term autoregression indicates that it is a regression of the variable against itself. Thus an autoregressive model of order $p$ can be written as

$$
y_{t}=c+\phi_{1} y_{t-1}+\phi_{2} y_{t-2}+\cdots+\phi_{p} y_{t-p}+e_{t},
$$

where $c$ is a constant and $e_{t}$ is white noise. This is like a multiple regression but with lagged values of $y_{t}$ as predictors. We refer to this as an $\mathrm{AR}(\mathrm{p})$ model.

\subsubsection{Moving average models}

Rather than use past values of the forecast variable in a regression, a moving average model uses past forecast errors in a regression-like model.

$$
y_{t}=c+e_{t}+\theta_{1} e_{t-1}+\theta_{2} e_{t-2}+\cdots+\theta_{q} e_{t-q},
$$

where $e_{t}$ is white noise. We refer to this as an MA(q) model. Of course, we do not observe the values of $e_{t}$, so it is not really regression in the usual sense.

\subsubsection{Non-seasonal ARIMA models}

If we combine differencing with autoregression and a moving average model, we obtain a nonseasonal ARIMA model. The full model can be written as

$$
y_{t}^{\prime}=c+\phi_{1} y_{t-1}^{\prime}+\cdots+\phi_{p} y_{t-p}^{\prime}+\theta_{1} e_{t-1}+\cdots+\theta_{q} e_{t-q}+e_{t},
$$


where $y_{t}^{\prime}$ is the differenced series (it may have been differenced more than once). The "predictors" on the right hand side include both lagged values of $y_{t}$ and lagged errors. We call this an ARIMA(p,d,q) model. Once we start combining components in this way to form more complicated models, it is much easier to work with the backshift notation. Then equation (2.3) can be written as

$$
\left(1-\phi_{1} B-\cdots-\phi_{p} B^{p}\right)(1-B)^{d} y_{t}=c+\left(1+\theta_{1} B+\cdots+\theta_{q} B^{q}\right) e_{t}
$$

where $B^{p} y_{t}=y_{t-p}$. Selecting appropriate values for $p, d$ and $q$ can be difficult. The auto.arima() function in $\mathrm{R}$ will do it automatically. This algorithm is also implemented in the $\mathrm{R}$ package forecast. The auto.arima() function in $\mathrm{R}$ uses a variation of the [8] algorithm that combines unit root tests, minimization of the AICc and MLE to obtain an ARIMA model.

The order of ARIMA model (i.e., the values of $p, d$ and $q$ ) is determined by Akaike's Information Criterion (AIC). The algorithm set upper bound on the order $p$ and $q$ at 5 , as it is almost impracticable to have the order $p$ or $q$ more than 5. Once the model order has been identified, we need to estimate the parameters $c, \phi_{1}, \cdots, \phi_{p}, \theta_{1}, \cdots, \theta_{q}$ by MLE. See [3] for detail.

\subsection{Dynamic linear models}

In recent decades, dynamic linear models, and state space models more generally, have increasingly become a focus of interest in time series analysis. Dynamic linear models (DLM) represent a special case of general state space models, being linear and Gaussian. These models are specified by means of two equations:

$$
\begin{aligned}
& y_{t}=H_{t} x_{t}+\varepsilon_{t} \\
& x_{t}=F_{t} x_{t-1}+\eta_{t},
\end{aligned}
$$

where $\varepsilon_{t} \sim N\left(0, \sigma_{\varepsilon}^{2}\right)$ and $\eta_{t} \sim N\left(0, \sigma_{\eta}^{2}\right)$ are two independent white noise sequences, $y_{t}$ denotes the observation at time $t$, and $x_{t}$ is the random vector of $k$ unobservable states of the system at time $t . H_{t}$ is a fixed vector and $F_{t}$ is a fixed state matrix. The usual features of a time series, such as trend and seasonality, can be modeled within this format. Sometimes, $H$ and $F$ are assumed to be independent of $t$, in which case the model is a time series DLM. [10] refer to such time series DLMs as multi disturbance or multiple source of error (MSOE) state space models, as the equations (2.4) and (2.5) have two independent source of errors, $\varepsilon_{t}$ and $\eta_{t}$.

When $\eta_{t}=g \varepsilon_{t}$ (where $g$ is a fixed vector of persistence parameters), the MSOE state space model becomes equivalent to the single source of error (SSOE) state space model introduced in Section 2.1.2. Table 2 (taken from [10]) shows the corresponding standard structural models from the two approaches for the local level and linear trend models. Note that although the common symbols $\ell, b$ and $\varepsilon$ are used to represent the level, slope and innovation respectively, their meanings differ between the two frameworks.

It is possible to represent an ARIMA model, whether univariate or multivariate, as a DLM (see Chapter 3 of [20]). However, in spite of the fact that an ARIMA model can formally be considered a DLM, the philosophies underlying the two classes of models are quite different: on the one hand, ARIMA models provide a black-box approach to data analysis, offering the possibility of forecasting future observations, but with a very limited interpretability of the fitted model; on the other hand, the DLM framework encourages the analyst to think in terms of easily interpretable, albeit unobservable, processes - such as trend and seasonal components - that drive the observed time series (for details, see Chapter 3 of [20]). 
Table 2. Comparison of local level and local linear trend models

\begin{tabular}{lll}
\hline Models MSOE models & SSOE models \\
\hline \multirow{2}{*}{ Level } & $y_{t}=\ell_{t-1}+\varepsilon_{t}$ & $y_{t}=\ell_{t-1}+\varepsilon_{t}$ \\
& $\ell_{t}=\ell_{t-1}+\eta_{t}$ & $\ell_{t}=\ell_{t-1}+\alpha \varepsilon_{t}$ \\
Trend & & \\
& $y_{t}=\ell_{t-1}+b_{t-1}+\varepsilon_{t}$ & $y_{t}=\ell_{t-1}+b_{t-1}+\varepsilon_{t}$ \\
& $\ell_{t}=\ell_{t-1}+b_{t-1}+\eta_{t}$ & $\ell_{t}=\ell_{t-1}+b_{t-1}+\alpha \varepsilon_{t}$ \\
& $b_{t}=b_{t-1}+\zeta_{t}$ & $b_{t}=b_{t-1}+\beta \varepsilon_{t}$
\end{tabular}

For DLM, estimation and forecasting can be obtained recursively by the well known Kalman filter [12]. This opens the way for estimation of any unknown parameter in the model (see for detail [6]). The R function StructTS in the R package stats is used to fit DLM. It uses the Kalman filter to implement the maximum likelihood approach to develop the estimates. Since all the data series considered are non-seasonal, therefore it selects the best model among non-seasonal DLM using AIC.

\section{Measures of forecast accuracy}

In the past, many studies have been conducted with the aim of identifying which method will provide the most accurate forecasts for a given class of time series data. In this section, the error measures used for drawing conclusions about the relative accuracies of models' forecast performances are discussed.

The Mean Square Error (MSE) is often used to compare the performances of forecasting models because of its computational convenience and theoretical relevance to statistics. However, it is scaledependent, and so should not be used to compare different series. The most widely used unit-free measure is the Mean Absolute Percentage Error (MAPE), and many books recommend its use $($ see $[2,5])$. However, one disadvantage of the MAPE is that it is relevant only for ratio-scaled data (i.e., data with a meaningful zero). [1] recommended the Median Relative Absolute Error (MdRAE) when few series are available, and the Median Absolute Percentage Error (MdAPE) otherwise. [15] discussed some problems with the RAE, and proposed an alternative measure called Symmetric Mean Absolute Percentage Error (sMAPE), a modification of the MAPE. [1] referred to this as the Unbiased Absolute Percentage Error (UAPE). However, [13] later commented on the sMAPE measure and showed that it actually penalizes low forecasts more than high forecasts, meaning that a more accurate name would be the Mean Asymmetric Absolute Percentage Error.

[9] proposed a new measure called the Mean Absolute Scaled Error (MASE), that is suitable for all three of the scenarios described earlier, and involves scaling the error based on the in-sample mean absolute error (MAE) from the naïve forecast method.

With so many measures of the forecast accuracy available, it is difficult to choose the most suitable one. In this paper, the following three commonly used forecast accuracy measures have been used to analyze the performances of the forecasts by applying them when forecasting the various time series. 
(i) Root Mean Squared Error (RMSE)

(ii) Mean Absolute Percentage Error (MAPE)

(iii) Mean Absolute Scaled Error (MASE)

These particular measures are chosen because several authors and practitioners believe that they are more reliable and sensitive to small changes than others (see [9]). Short descriptions of these accuracy measures are given below.

\section{(i) RMSE}

The root mean squared error (RMSE) is defined as follows:

$$
\mathrm{RMSE}=\sqrt{\frac{1}{n} \sum_{t=1}^{n}\left(Y_{t}-F_{t}\right)^{2}},
$$

where $Y_{t}$ is the observed value, $F_{t}$ is the forecast (predicted) value at time $t$, and $n$ is the length of the data series.

(ii) $M A P E$

First, a relative or percentage error is defined as

$$
\mathrm{PE}_{t}=\left(\frac{Y_{t}-F_{t}}{Y_{t}}\right) \times 100,
$$

where $Y_{t}$ is the actual value and $F_{t}$ is the forecast value at time $t$. Then, the MAPE is defined as the average of the absolute values of PE, as follows (for more details, see Makridakis et al., 1998):

$$
\mathrm{MAPE}=\frac{1}{n} \sum_{t=1}^{n}\left|\mathrm{PE}_{t}\right| .
$$

(iii) MASE

[9] proposed a new but related idea that is suitable for all three of the scenarios described earlier. This scaled error is defined as

$$
q_{t}=\frac{Y_{t}-F_{t}}{\frac{1}{n-1} \sum_{i=2}^{n}\left|Y_{i}-Y_{i-1}\right|},
$$

which is clearly independent of the scale of the data. A scaled error is less than one if it arises from a better forecast than the average one-step naïve forecast computed in-sample. Conversely, it is greater than one if the forecast is worse than the average one-step naïve forecast computed in-sample. The Mean Absolute Scaled Error (MASE) is simply

$$
\text { MASE }=\text { mean }\left(\left|q_{t}\right|\right) .
$$

The in-sample mean absolute error (MAE) is used in the denominator as it is always available and effectively scales the errors. In contrast, the out-of-sample MAE for the naïve method can be based on very few observations and is therefore more variable. The only situation in which these measures would be infinite or undefined is when all historical observations are equal. 


\section{Empirical analysis}

In this section, the exponential smoothing method, the dynamic linear model and the Box-Jenkins method are applied to some real agriculture time series data in order to demonstrate the differences between the methods/models in an application. The time series used are wheat production (WP), rice production (RP), cotton production (CP) and pulses production (PP). All of the data sets are annual, covering the period 1961 to 2012 for wheat and rice, and the period 1971 to 2011 for cotton and pulses. The data have been collected from various issues of the Agricultural Statistics of Pakistan, published by the Ministry of Food and Agriculture (Economic Wing), government of Pakistan, and Pakistan Economic Surveys, published by the Economic Advisor wing, Finance Division, government of Pakistan. The time series are plotted in Figure 1.
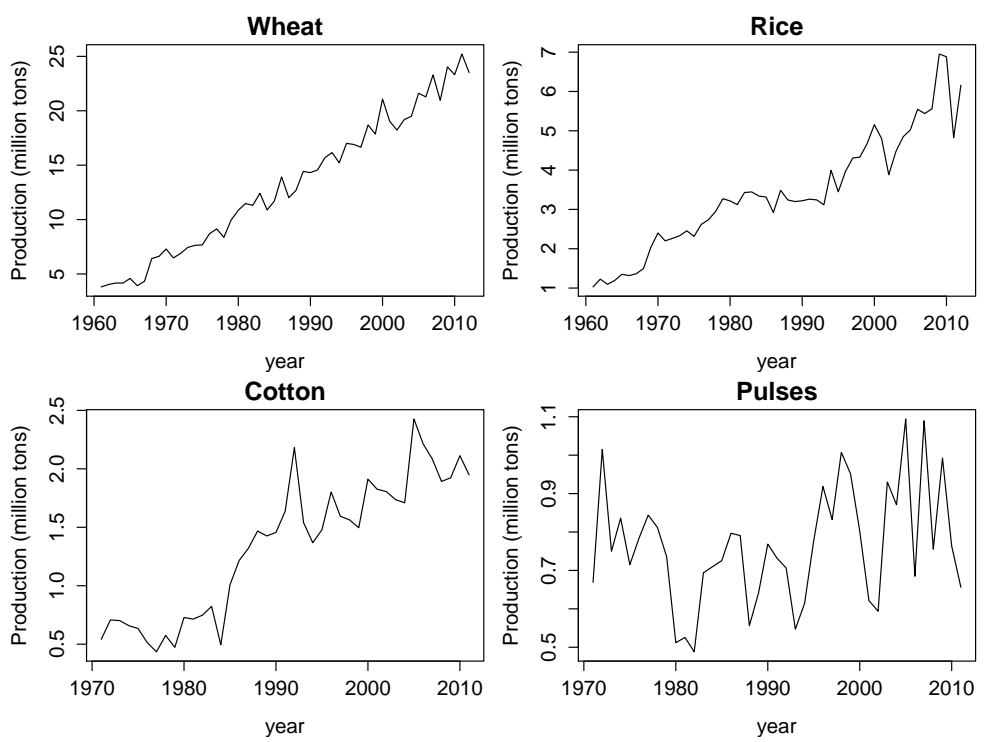

Fig. 1. Time series data

Agriculture is the backbone of the Pakistan's economy, and plays an important role in the betterment of the large proportion of the population that live in rural areas in particular, and the overall economy in general. The growing population of Pakistan is a real point of concern that needs to be addressed by proper and timely planning for a food supply. Regarding food grain crops, wheat and rice are the main staple foods of the people of Pakistan, as well as being the most important parts of the agriculture sector.

To enable a forecast comparison, each of the time series (Figure 1) is divided into two parts, a training set (comprising $80 \%$ of the data) and a test set (comprising $20 \%$ of the data). That is, we hold back the last $20 \%$ of the data, called the test set (we may call it future sample paths), and fit the model on the rest of the data, called the training set. The best selected exponential smoothing model, the best selected DLM and the best selected ARIMA model are then fitted to the training set using maximum likelihood estimation.

In order to compare the performances of the models, we have plotted the forecasts of wheat, rice, cotton and pulses production in Figures 2-5, respectively. Top panel: forecasts from the best selected exponential smoothing model. Middle panel: forecasts from the best selected ARIMA. 
Bottom panel: forecasts from the best selected DLM model. The forecasts are based on models fitted to the data up to 2003. The dark (light) shaded region indicates $95 \%$ (80\%) prediction intervals. The actual values in the forecast period are also shown. Overall, the exponential smoothing method tends to perform well, as it is generally the best (for the wheat and rice time series) or second-best (for the cotton and pulses time series) in terms of forecast accuracies, and gives more reasonable forecasts than its DLM and ARIMA counterparts. The performance of DLM is notable, as it outperforms the ARIMA method in all cases except for the pulses series. From Figure 2, it can be seen that the forecasts from the three methods all look quite similar, underestimating the actual values of wheat production. However, the measures of forecasting accuracy (see Table 3) suggest that the exponential smoothing method performs better than its counterparts. Moreover, the actual values in the forecast period are well within the $95 \%$ prediction intervals obtained via exponential smoothing.
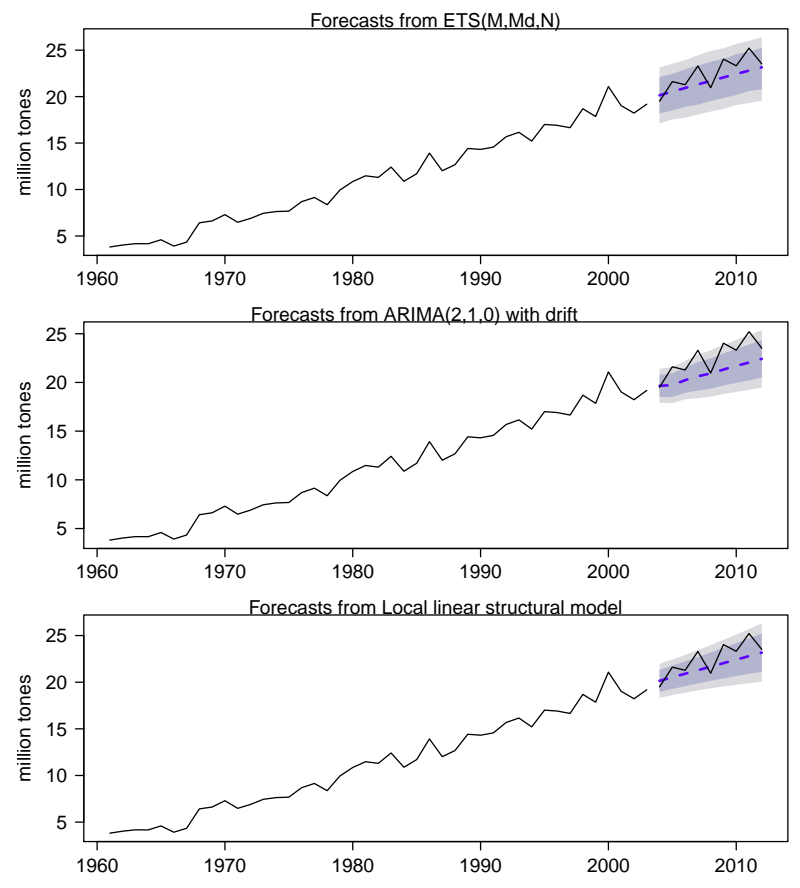

Fig. 2. Historical data (solid line) and forecasts (dashed line) for the wheat production time series.

The forecasts and prediction intervals produced by the three methods for the rice time series are shown in Figure 3. The forecasts obtained from the various methods look quite different; in particular, the prediction intervals are much wider in the case of exponential smoothing than for the other methods. This is because the prediction intervals produced by ES reflect the uncertainty associated with the data set, especially at the end of the training set, while the ARIMA and DLM methods seem to fail to pick it up properly. Furthermore, according to the forecast accuracy measures, the ES method again performs well relative to the other techniques for the rice time series data (see Table 3).

Figure 4 shows that the ES and ARIMA methods are both unable to produce very good forecasts for the cotton time series relative to the DLM, which seems to pick up some form of a trend from the training set. According to the forecast accuracy measures, the DLM takes the lead for the cotton 

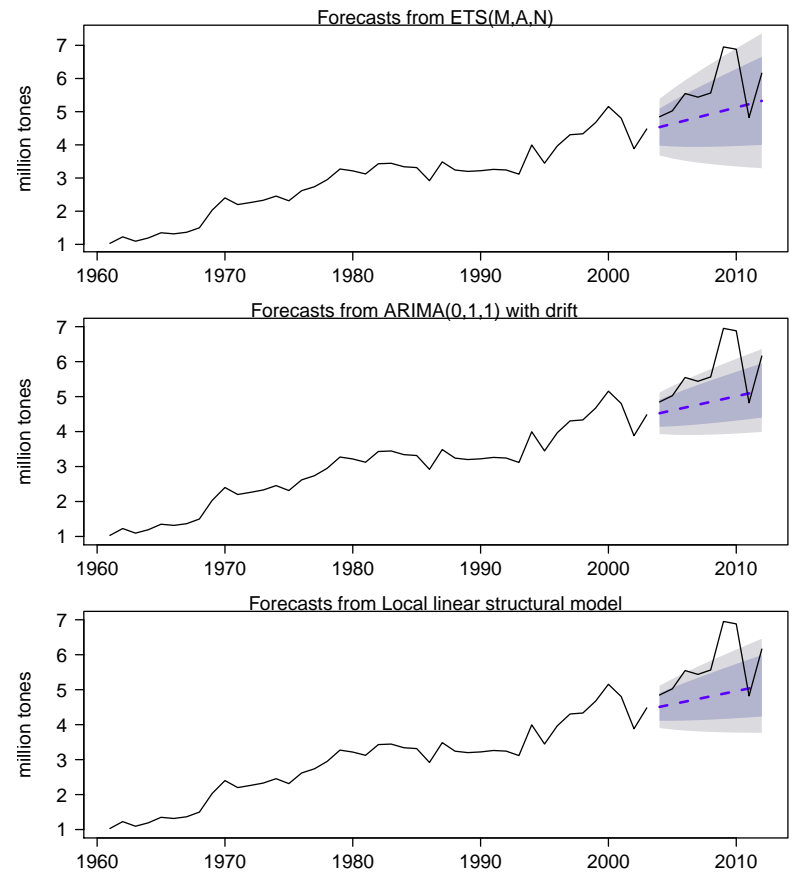

Fig. 3. Historical data (solid line) and forecasts (dashed line) for the rice production time series.

time series, although the exponential smoothing method again beats the Box-Jenkins ARIMA technique. Only for the pulses production data (Figure 5), where strong stationarity is very prominent, the Box-Jenkins ARIMA technique perform better than the other methods, with ES again standing second-best.

The various measures of forecast accuracy, namely the root mean squared error (RMSE), mean absolute percentage error (MAPE) and mean absolute scaled error (MASE), have also been calculated for all data sets over the forecast period (test set or future sample paths) for each model. According to these accuracy measures (Table 3), the exponential smoothing method comes out as the best for the wheat and rice time series, and second-best for the cotton and pulses time series. The DLM performs best for the cotton time series, while the Box-Jenkins ARIMA technique is best for the pulses time series, where strong stationarity is very prominent. While these are only few empirical examples, making it hard to draw general conclusions, the evidence is consistent with Figures 2 to 5 in suggesting that the exponential smoothing method performs better than either the DLM or Box-Jenkins ARIMA method.

\section{Conclusions}

The main objective of any forecasting method is to make precise predictions of future random events. This paper has assessed different forecasting methods based on their out-of-sample forecast accuracy measures. Three methods, Box-Jenkins' ARIMA, exponential smoothing (ES) and dynamic linear models (DLM), have been used to produce predictions for four important crops in Pakistan, that play important roles in Pakistan's economy. 

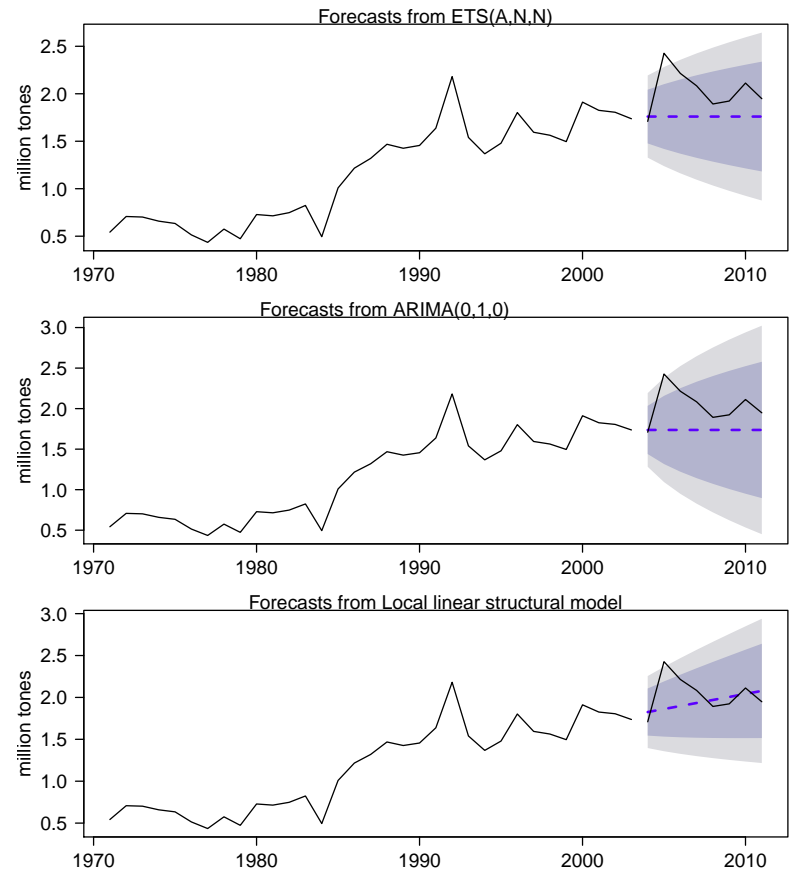

Fig. 4. Historical data (solid line) and forecasts (dashed line) for the cotton production time series.
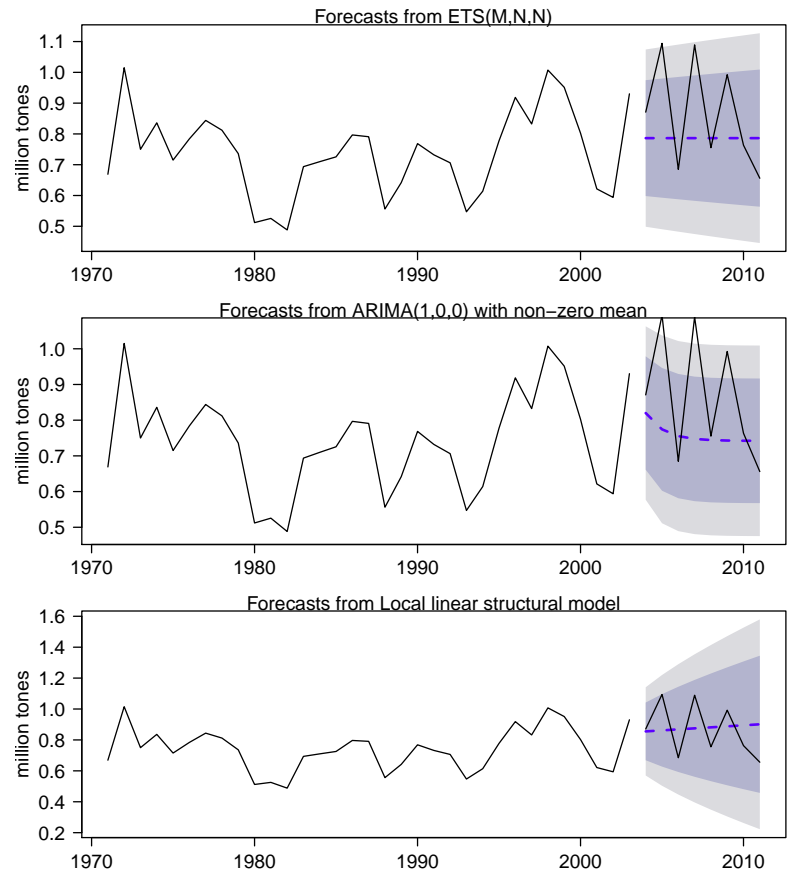

Fig. 5. Historical data (solid line) and forecasts (dashed line) for the pulses production time series. 
Table 3. Measures of forecast accuracy for each model

\begin{tabular}{llcccc}
\hline Measure & Method & Wheat & Rice & Cotton & Pulses \\
\hline \hline \multirow{3}{*}{ MASE } & & & & & \\
& ES & 1.302 & 3.472 & 1.759 & 1.285 \\
& ARIMA & 1.797 & 3.678 & 1.864 & 1.245 \\
& DLM & 1.308 & 3.788 & 1.138 & 1.356 \\
MAPE & ES & 5.004 & 14.062 & 13.544 & 16.030 \\
& ARIMA & 6.830 & 14.825 & 14.352 & 14.902 \\
& DLM & 5.026 & 15.264 & 8.749 & 18.914 \\
& & & & & \\
RMSE & ES & 1366 & 1018 & 347 & 182 \\
& ARIMA & 1913 & 1086 & 366 & 193 \\
& DLM & 1374 & 1119 & 248 & 173 \\
\hline
\end{tabular}

Empirical comparisons have been performed using various different forecast accuracy measures, which have suggested that the exponential smoothing method performs better (either best or secondbest in terms of the forecast accuracy measures) than its competitors, the DLM and the Box-Jenkins ARIMA. The DLM performs best for the cotton time series, followed by ES, while ARIMA does better for pulses, followed by ES. These forecasts may be helpful in determining prices of these four commodities in advance for the farmers and financial institutions.

\section{References}

[1] J. S. Armstrong and F Collopy, Error measures for generalizing about forecasting methods: empirical comparison, International Journal of Forecasting 08 (1992) 69-80.

[2] B.L. Bowerman, R.T. OConnell and A.B. Koehler, Forecasting, time series and regression: an applied approach, (Thomson Brooks/Cole, Belmont CA, 2004).

[3] G.E.P. Box, G. Jenkins and G.C. Reinsel, Time series analysis: forecasting and control, 4th Ed (Hoboken, N.J. : John Wiley, 2008).

[4] R.G. Brown, Statistical forecasting for inventory control, 4th edn (McGraw-Hill, New York, 1959).

[5] J.E. Hanke and A.G. Reitsch, Business forecasting, 5th edn (Prentice-Hall: Englewood Cliffs, NJ, 1995).

[6] A.C. Harvey, Forecasting, structural time series models and the Kalman filter, (Cambridge University Press, Cambridge, 1989).

[7] R.J. Hyndman, G. Athanasopoulos, S. Razbash, D. Schmidt, Z. Zhou and Y Khan, forecast: Forecasting functions for time series and linear models., $R$ package version, URL: http://CRAN.Rproject.org/package=forecast $\mathbf{4 . 0 4}$ (2013).

[8] R.J. Hyndman, and Y Khandakar, Automatic time sereis forecasting: the forecast package for r, Journal of Statistical Software 27(3) (2008).

[9] R.J. Hyndman, and A.B. Koehler, Another look at measures of forecast accuracy, International Journal of Forecasting 22(4) (2006) 679-688.

[10] R.J. Hyndman, A.B. Koehler, J.K. Ord and R.D. Snyder, Forecasting with exponential smoothing: The state space approach, (Springer-Verlag: Berlin Heidelberg, 2008).

[11] R.J. Hyndman, A.B. Koehler, R.D. Snyder and S. Grose, A state space framework for automatic forecasting using exponential smoothing methods, International Journal of Forecasting 18(3) (2002) 439454. 
[12] R.E. Kalman, A new approach to linear filtering and prediction problems, Journal of Basic Engineering (Series D) 82(1) (1960) 35-45.

[13] R.E. Kalman, The asymmetry of the sAPE measure and other comments on the M3-competition, International Journal of Forecasting 17 (2001) 537-584.

[14] S. Makridakis and M. Hibon, The M3-competition: results, conclusions and implications, International Journal of Forecasting 16 (2000) 451-476.

[15] S. Makridakis, Accuracy measures: theoretical and practical concerns, International Journal of Forecasting 9 (1993) 527-529.

[16] S. Makridakis, A. Anderson, R.Carbone, R. Fildes, M. Hibon, R.L.J. Newton, E. Parzen and R. Winkler, The accuracy of extrapolation (time series) methods: results of a forecasting competition, Journal of Forecasting 1 (1982) 111-153.

[17] S. Makridakis, S.C. Wheelwright and R.J. Hyndman, Forecasting: methods and applications, 3rd edn (John Wiley and Sons, New York, 1998).

[18] J.K. Ord, A.B. Koehler and R.D. Snyder, Estimation and prediction for a class of dynamic nonlinear statistical models, Journal of American Statistical Association 92 (1997) 1621-1629.

[19] C.C. Pegels, Exponential smoothing: some new variations, Management Science 12 (1969) 311-315.

[20] G. Petris, S. Petrone and R. Campagnoli, Dynamic Linear Models with R, (Springer, 2009).

[21] R Core Team (2013), R: A Language and Environment for Statistical Computing, $R$ Foundation for Statistical Computing, (Vienna, Austria, URL: http://www.R-project.org/, 2009).

[22] J.W. Taylor, Exponential smothing with damped multiplicative trend, International Journal of Forecasting 19 (2003) 715-725. 